\title{
SHORT COMMUNICATIONS \\ REMARKS ON RELATIONS BETWEEN PENALTY AND MIXED FINITE ELEMENT METHODS FOR A CLASS OF VARIATIONAL INEQUALITIES
}

\author{
NOBORU KIKUCHI \\ The University of Michigan, Ann Arbor, Michigan, U.S.A. \\ AND \\ YOUNG JOON SONG \\ Korea Military Academy, Seoul, Korea

\section{INTRODUCTION}

This short communication is intended to describe a characterization of a penalty-finite element approximation for a variational inequality. Particularly, we will show that the solution $u_{\varepsilon}^{h}$ of the penalty-finite element approximation and the contact pressure $p_{\varepsilon}^{h}$ converge to the solution $\left\{u^{h}, p^{h}\right\}$ of a mixed formulation of the variational inequality.

Theories of equivalence between penalized and mixed finite element methods have been studied by Malkus, ${ }^{1}$ Hughes, ${ }^{2}$ Malkus and Hughes ${ }^{3}$ and Bercovier ${ }^{4}$ for the Stokes problem, and by Lee and Pian ${ }^{5}$ for plate and shell problems. The present study is an extension of these works, which are concerned with constraints represented by equations, to penalty methods resolving constraints given by inequalities.

The first result of the present paper is that the penalized solution $u_{\varepsilon}^{h}$ converges to the one of the variational inequality in the discrete form. The second is the convergence of $\left\{u_{\varepsilon}^{h}, p_{\varepsilon}^{h}\right\}$ to the solution $\left\{u^{h}, p^{h}\right\}$ of a mixed formulation of the variational inequality. The last result is an error estimate of approximations with respect to the penalty parameter $\varepsilon$, for a fixed mesh size $h$ of the finite element model.

\section{A UNILATERAL PROBLEM}

Suppose that the variational inequality

$$
u \in K: a(u, v-u) \geqslant f(v-u), \quad \forall v \in K
$$

is given together with the constrained set

$$
K=\{v \in V: v-s \leqslant 0 \text { in } \Omega\}
$$

where $V$ is a closed subspace of the Sobolev space $H^{m}(\Omega), m \geqslant 0$, and $\Omega$ is a bounded open domain in $R^{3}$. Suppose that the boundary $\Gamma$ of $\Omega$ is smooth enough. The variational inequality (1) represents the obstacle problem of an elastic membrane, Signorini's problem of a plate or a linearly elastic body, and so on, see Duvaunt and Lions. ${ }^{6}$

Suppose that the bilinear form $a(.,$.$) is coercive and continuous on V$ and the linear form $f($. is continuous on $V$, i.e.

$$
a(u, u) \geqslant m\|u\|_{m}^{2} \quad a(u, v) \leqslant M\|u\|_{m}\|v\|_{m} \quad f(v) \leqslant\|f\|_{m}^{*}\|v\|_{m}
$$


where $\left\|_{.}\right\|_{m}$ is the norm of $H^{m}(\Omega)$, and $\|.\|_{m}^{*}$ is its dual norm. Let the distance function $s$ in the constrained set be an element of $H^{m}(\Omega)$, i.e.

$$
s \in H^{m}(\Omega)
$$

\section{A PENALTY-FINITE ELEMENT APPROXIMATION}

One of practical methods of approximation of the variational inequality (1) is a penalty-finite element approximation.

Let $V_{h}$ be the finite element approximation of the space $V$. Let $b_{h}$ be an approximation of the penalty function to the constraint given by

$$
b_{h}\left(v^{h}\right)=\left(v^{h}-s\right)(x)^{+}
$$

where $g^{+}=\operatorname{Max}(0,, g)$ for $g \in R$. Let $(.,$.$) be the L^{2}(\Omega)$-inner product defined by

$$
(f, g)=\int_{\Omega} f g \mathrm{~d} x
$$

and let $I_{G}$ be the operation of numerical integration. Then, the penalty-finite element approximation of (1) is given by

$$
u_{\varepsilon}^{h} \in V_{h}: a\left(u_{\varepsilon}^{h}, v^{h}\right)+\frac{1}{\varepsilon} I_{G}\left(b_{h}\left(u_{\varepsilon}^{h}\right), v^{h}\right)=f\left(v^{h}\right), \quad \forall v^{h} \in V_{h}
$$

where $\varepsilon$ is a penalty parameter and

$$
I_{G}\left(b_{h}\left(u_{\varepsilon}^{h}\right), v^{h}\right)=\sum_{e=1}^{E} \sum_{i=1}^{G} w_{i} b_{h}\left(u_{\varepsilon}^{h}\right)\left(x_{i}^{e}\right) v^{h}\left(x_{i}^{e}\right)
$$

Here $E$ is the total number of elements, $G$ is the total number of integration points within an element, $w_{i}$ and $x_{i}^{e}$ are weights and points of numerical integration, and $i=1,2, \ldots, G$, $e=1,2, \ldots, E$. The reason why the rule of numerical integration $I_{G}$ is applied to the term of penalty is that the exact integration of it is impossible because of the special nonlinearity of the penalty function $b_{n}\left(u_{\varepsilon}^{h}\right)$.

We first show that the sequence $u_{\varepsilon}^{h}$ of the solution of the penalized formulation (7) converges to a solution of an approximation of the variational inequality (1), as $\varepsilon$ goes to zero.

Theorem 1. Suppose that (3) and (4) hold. Then the solution $u_{\varepsilon}^{h}$ of (3) is uniformly bounded in $\varepsilon$, and the sequence $u_{\varepsilon}^{h}$ converges to a solution $u^{h}$ of the variational inequality

$$
\left\{\begin{array}{l}
u^{h} \in K_{h}: a\left(u^{h}, v^{h}-u^{h}\right) \geqslant f\left(v^{h}-u^{h}\right), \quad \forall v^{h} \in K_{h} \\
K_{h}=\left\{v^{h} \in V_{h}:\left(v^{h}-s\right)\left(x_{i}^{e}\right) \leqslant 0, i=1, \ldots, G, e=1, \ldots, E\right\}
\end{array}\right.
$$

as the penalty parameter $\varepsilon$ goes to zero.

(Proof) (Uniform Boundedness) Let $v^{h} \in K_{h}$. From (7),

$$
a\left(u_{\varepsilon}^{h}, v^{h}-u_{\varepsilon}^{h}\right)+\frac{1}{\varepsilon} I_{G}\left(b_{h}\left(u_{\varepsilon}^{h}\right), v^{h}-u_{\varepsilon}^{h}\right)=f\left(v^{h}-u_{\varepsilon}^{h}\right)
$$

Since $b_{h}\left(v^{h}\right)=0$.

$$
\begin{aligned}
I_{G}\left(b_{h}\left(u_{\varepsilon}^{h}\right), v^{h}-u_{\varepsilon}^{h}\right) & =-I_{G}\left(b_{h}\left(v^{h}\right)-b_{h}\left(u_{\varepsilon}^{h}\right), v^{h}-u_{\varepsilon}^{h}\right) \\
& \leqslant-I_{G}\left(\left(v^{h}-s\right)^{+}-\left(u_{\varepsilon}^{h}-s\right)^{+},\left(v^{h}-s\right)^{+}-\left(u_{\varepsilon}^{h}-s\right)^{+}\right) \leqslant 0
\end{aligned}
$$


Thus we have

$$
a\left(u_{\varepsilon}^{h}, v^{h}-u_{\varepsilon}^{h}\right) \geqslant f\left(v^{h}-u_{\varepsilon}^{h}\right), \quad \forall v^{h} \in K_{h}
$$

This inequality also implies the estimate

$$
\left\|u_{\varepsilon}^{h}\right\|_{m} \leqslant C, \quad \varepsilon>0
$$

(Convergence) Since $V_{h}$ is a (closed) finite dimensional subspace of a reflexive Banach space $H^{m}(\Omega)$, there exists a subsequence of $u_{\varepsilon}^{h}$, still denoted by $u_{\varepsilon}^{h}$, which converges to $u^{h}$ in $V_{h}$. Passing to the limit $\varepsilon \rightarrow 0$ in (12), we have

$$
a\left(u^{h}, v^{h}-u^{h}\right) \geqslant f\left(v^{h}-u^{h}\right), \quad \forall v^{h} \in K_{h}
$$

Thus we need show only that $u^{h} \in K_{h}$. Putting $v^{h}-u_{\varepsilon}^{h}$ in (7),

$$
a\left(u_{\varepsilon}^{h}, v^{h}-u_{\varepsilon}^{h}\right)+\frac{1}{\varepsilon} I_{G}\left(\left(u_{\varepsilon}^{h}-s\right)^{+}, v^{h}-u_{\varepsilon}^{h}\right)=f\left(v^{h}-u_{\varepsilon}^{h}\right)
$$

If $v^{h} \in K_{h}$, i.e. if $v^{h}-s \leqslant 0$ at $x_{i}^{e}$,

$$
I_{G}\left(\left(u_{\varepsilon}^{h}-s\right)^{+}, v^{h}-u_{\varepsilon}^{h}\right)=I_{G}\left(\left(u_{\varepsilon}^{h}-s\right)^{+}, v^{h}-s-\left(u_{\varepsilon}^{h}-s\right)\right) \leqslant-I_{G}\left(\left(u_{\varepsilon}^{h}-s\right)^{+},\left(u_{\varepsilon}^{h}-s\right)^{+}\right)
$$

Then we have the inequality

$$
a\left(u_{\varepsilon}^{h}, v^{h}-u_{\varepsilon}^{h}\right)-\frac{1}{\varepsilon} I_{G}\left(\left(u_{\varepsilon}^{h}-s\right)^{+},\left(u_{\varepsilon}^{h}-s\right)^{+}\right) \geqslant f\left(v^{h}-u_{\varepsilon}^{h}\right)
$$

i.e.

$$
I_{G}\left(\left(u_{\varepsilon}^{h}-s\right)^{+},\left(u_{\varepsilon}^{h}-s\right)^{+}\right) \leqslant \varepsilon\left(a\left(u_{\varepsilon}^{h}, v^{h}\right)+f\left(u_{\varepsilon}^{h}-v^{h}\right)\right)
$$

Since $u_{\varepsilon}^{h}$ is uniformly bounded in $\varepsilon$ and since $v^{h}$ is an arbitrary but fixed element in $K_{h}$, we can conclude that

$$
\left|\left(u_{\varepsilon}^{h}-s\right)\left(x_{i}^{e}\right)^{+}\right|^{2} \leqslant C \varepsilon, \quad \text { then } \quad\left(u^{h}-s\right)\left(x_{i}^{e}\right)^{+}=0
$$

Therefore $u^{h} \in K_{h}$.

We have shown that the penalty solution $u_{e}^{h}$ converges to a solution $u^{h}$ of the variational inequality (9) which may be considered as an approximation of (1). We now explore substance of (9). To this end, we first find a meaning of the set $K_{h}$ in which the contact condition is satisfied pointwise.

Let $q^{h}$ be an arbitrary element of the piecewise polynomial space $Q_{h}$ such that

$$
\left(q^{h}, v^{h}\right)=I_{G}\left(q^{h}, v^{h}\right), \quad \forall v^{h} \in V_{h}
$$

If $G=3$ and $V_{h}$ consists of piecewise cubic polynomials in the one-dimensional domain $\Omega=(0,1), q^{h}$ is a piecewise quadratic polynomial. Then, if $s \in V_{h}$,

$$
v^{h} \in K_{h} \quad \text { if and only if }\left(q^{h}, v^{h}-s\right) \geqslant 0, \quad \forall q^{h} \in N_{h}
$$

where

$$
N_{h}=\left\{q^{h} \in Q_{h}: q^{h}\left(x_{i}^{e}\right) \leqslant 0, i=1, \ldots, G, e=1, \ldots, E\right\}
$$

Using the dual $N_{h}$ of the constrained set $K_{h}$, we can characterize the set $K_{h}$ by

$$
K_{h}=\left\{v^{h} \in V_{h}:\left(q^{h}, v^{h}-s\right) \geqslant 0, \quad \forall q^{h} \in N_{h}\right\}
$$


It is now clear that $q^{h}$ is the Lagrangian multiplier to the constraint $v^{h}-s \leqslant 0$, and represents the approximation of the contact pressure due to contact, physically. We will find relations between the penalty and Lagrangian multiplier (or mixed) methods, more precisely. Define $p^{h} \in Q_{h}$ by

$$
p_{\varepsilon}^{h}\left(x_{i}^{e}\right)=-\frac{1}{\varepsilon}\left(u_{\varepsilon}^{h}-s\right)\left(x_{i}^{e}\right)^{+}
$$

Then, by (14) and (18), the penalty form (7) can be written as

$$
a\left(u_{\varepsilon}^{h}, v^{h}\right)-\left(p_{\varepsilon}^{h}, v^{h}\right)=f\left(v^{h}\right), \quad \forall v^{h} \in V_{h}
$$

The form (19) suggests that $p_{\varepsilon}^{h} \in Q_{h}$ is an approximation of the contact pressure.

Theorem 2. Suppose that (14) and $s \in V_{h}$ hold. Further suppose that

$$
C_{h}>0: C_{h}\left\|q^{h}\right\|_{0} \leqslant \sup _{v^{h} \in V_{h}} \frac{\left(q^{h}, v^{k}\right)}{\left\|v^{h}\right\|_{m}}, \quad \forall q^{h} \in Q_{h}
$$

Then $p_{\varepsilon}^{h} \in Q_{h}$, defined by (18), is uniformly bounded in $\varepsilon$, and converges to $p^{h} \in Q_{h} \cap N_{h}$ which satisfies

$$
a\left(u^{h}, v^{h}\right)-\left(p^{h}, v^{h}\right)=f\left(v^{h}\right), \quad \forall v^{h} \in V_{h} \quad\left(q^{h}-p^{h}, u^{h}-s\right) \geqslant 0, \quad \forall q^{h} \in N_{h}
$$

(Proof) Using (19) and (20),

$$
C_{h}\left\|p_{\varepsilon}^{h}\right\|_{0} \leqslant \sup _{v^{h} \in V_{h}} \frac{a\left(u_{s}^{h}, v^{h}\right)-f\left(v^{h}\right)}{\left\|v^{h}\right\|_{m}} \leqslant M\left\|u_{\varepsilon}^{h}\right\|_{m}+\|f\|_{m}^{*}
$$

Since $u_{\varepsilon}^{h}$ is uniformly bounded in $\varepsilon, p_{\varepsilon}^{h}$ is also uniformly bounded in $L^{2}(\Omega)$-sense. Then there is a subsequence of $p_{\varepsilon}^{h}$, still denoted by $p_{\varepsilon}^{h}$, which converges to $p^{h}$ in $Q_{h}$. Passing to the limit $\varepsilon \rightarrow 0$ in (19) yields (21) ${ }_{1}$. We will show (21) 2 . By (18),

$$
\left(q^{h}-p_{\varepsilon}^{h}, u_{\varepsilon}^{h}-s\right)=\left(q^{h}, u_{\varepsilon}^{h}-s\right)+\frac{1}{\varepsilon} I_{G}\left(\left(u_{\varepsilon}^{h}-s\right)^{+}, u_{\varepsilon}^{h}-s\right) \geqslant\left(q^{h}, u_{\varepsilon}^{h}-s\right)
$$

Passing to the limit $\varepsilon \rightarrow 0$ in the above for $q^{h} \in N_{h}$,

$$
\left(q^{h}-p^{h}, u^{h}-s\right) \geqslant\left(q^{h}, u^{h}-s\right) \geqslant 0, \quad \forall q^{h} \in N_{h}
$$

since $u^{h} \in K_{h}$.

Similar results of convergence for the penalty-finite element approximation can be also obtained for the case that a proper space $Q_{h}$ of the contact pressure, satisfying the relation (14), does not exist. The corresponding convergence theorem for such cases is stated by the mixed formulation (21) with the operation of numerical integration $I_{G}$, see Kikuchi. ${ }^{7}$

So far we could characterize the penalty-finite element approximation (7) as an approximation of a mixed (Lagrangian multiplier) formulation to the variational inequality (1) under the additional condition (20), which is called the discrete L.B.B. condition in the literature of mixed finite element methods.

We finally give rates of convergence of $u_{\varepsilon}^{h}$ and $p_{\varepsilon}^{h}$ for the penalty-finite element approximation. 
Theorem 3. Same conditions in Theorem 2 hold. Then

$$
\left\|u^{h}-u_{e}^{h}\right\|_{1} \leqslant(M / m)\left\|p^{h}\right\|_{0} \varepsilon / C_{h}, \quad\left\|p^{h}-p_{e}^{h}\right\|_{0} \leqslant M\left\|u^{h}-u_{e}^{h}\right\|_{1} / C_{h}
$$

where $C_{h}$ is the constant in (20).

(Proof) From (19) and (21),

$$
a\left(u^{h}-u_{\varepsilon}^{h}, v^{h}\right)=\left(p^{h}-p_{\varepsilon}^{h}, v^{h}\right)
$$

Taking $v^{h}=u^{h}-u_{e}^{h}$ in (23) yields

$$
\begin{aligned}
a\left(u^{h}-u_{\varepsilon}^{h}, u^{h}-u_{\varepsilon}^{h}\right) & =\left(p^{h}-p_{\varepsilon}^{h}, u^{h}-u_{\varepsilon}^{h}\right) \\
& =\left(p^{h}-p_{\varepsilon}^{h}, u^{h}-s\right)+\left(p^{h}-p_{\varepsilon}^{h},\left(u_{\varepsilon}^{h}-s\right)^{-}\right)-\left(p^{h}-p_{\varepsilon}^{h},\left(u_{\varepsilon}^{h}-s\right)^{+}\right) \\
& \leqslant-\left(p^{h}-p_{\varepsilon}^{h}, p^{h}-p_{\varepsilon}^{h}\right) \varepsilon+\left(p^{h}-p_{\varepsilon}^{h}, p^{h}\right) \varepsilon
\end{aligned}
$$

since (14), (18) and (21). Here $v^{-}=v^{+}-v$.

Applying (3), we have

$$
m\left\|u^{h}-u_{\varepsilon}^{h}\right\|_{1}^{2} \leqslant\left\|p^{h}-p_{\varepsilon}^{h}\right\|_{0}\left\|p^{h}\right\|_{0} \varepsilon
$$

On the other hand, from (23)

$$
C_{h}\left\|p^{h}-p_{\varepsilon}^{h}\right\|_{0} \leqslant M\left\|u^{h}-u_{\varepsilon}^{h}\right\|_{1}
$$

Here (3) and (20) have been applied. Therefore, (22) follows from (24) and (25).

\section{ACKNOWLEDGEMENTS}

The authors are grateful to Prof. J. Tinsley Oden for his discussions. During this work, the authors were supported by AFOSR F-49620-78-C-0083.

\section{REFERENCES}

1. D.S. Malkus, 'A finite element displacement model valid for any value of the compressibility', Int. J. Solids Struct. 12, $731-738(1976)$.

2. T. J. R. Hughes, 'Equivalence of finite elements for nearly incompressible elasticity', $L B L 5237$, University of California (Aug. 1976).

3. D. S. Malkus and T. J. R. Hughes, 'Mixed finite element methods-reduced and selective integration techniques: a unified concepts', Comp. Meth. Appl. Mech. Engng. 12, 63-81 (1978).

4. M. Bercovier, 'Pertubation of a mixed variational problem; application to mixed finite element methods', $R A I R O$, Numer. Anal. 12, 211-236 (1978).

5. S. W. Lee and T. H. H. Pian, 'Improvements of plate and shell finite elements by mixed formulations', $A$ IAA/ASME 18th Structures, Structural Dynamics and Material Conference, San Diego, California (21-23 March, 1977).

6. G. Duvaut and J. L. Lions, Inequalities in Mechanics and Physics, Springer-Verlag, Berlin, 1976.

7. N. Kikuchi, 'Convergence of a penalty-finite element approxtmation for an obstacle problem', in review. 